\title{
Tangence
}

\section{Raconter des histoires de père en fille : portraits d'Yves et de Marie José Thériault}

\section{Renald Bérubé}

Numéro 62, avril 2000

Parentèle

URI : https://id.erudit.org/iderudit/008175ar

DOI : https://doi.org/10.7202/008175ar

Aller au sommaire du numéro

Éditeur(s)

Presses de l'Université du Québec

ISSN

0226-9554 (imprimé)

1710-0305 (numérique)

Découvrir la revue

Citer cet article

Bérubé, R. (2000). Raconter des histoires de père en fille : portraits d'Yves et de Marie José Thériault. Tangence, (62), 84-99. https://doi.org/10.7202/008175ar d'utilisation que vous pouvez consulter en ligne.

https://apropos.erudit.org/fr/usagers/politique-dutilisation/ 


\section{Raconter des histoires de père en fille: portraits d'Yves et de Marie José Thériault Renald Bérubé, Université du Québec à Rimouski}

Quelqu'un se mit à faire une chanson. Il parlait d'Agaguk, grand chasseur. Et il chantait aussi Tayaout qui serait bien le plus grand chasseur des temps, fils d'un tel père.

La chanson dura longtemps. Agaguk s'en saoûlait.

$$
\text { Y. Thériault, Agaguk }{ }^{1}
$$

L'histoire de Didem-au-Petit-Serpent et du musicien d'Alep traversa l'univers des Croyants comme une traînée de poudre. Les chanteurs l'ont chantée. Les conteurs l'ont contée. Les poètes l'ont mise en vers qu'on a brodés sur des bannières et suspendus dans les mosquées.

M. J. Thériault, "Histoire de Didem-au-PetitSerpent et du musicien d'Alep", dans Portraits d'Elsa et autres histoires ${ }^{2}$

Homer adossa sa chaise au mur de la cuisine et se mit à lui raconter l'histoire du dernier colonel Cary, qui avait légué l'île à l'Institut. Et qui était une femme.

Marian Engel, Ours (trad. de M. J. Thériault) ${ }^{3}$

1 Yves Thériault, Agaguk, Montréal, l'Homme, [1958] 1963, p. 134.

2 Marie José Thériault, Portraits d'Elsa et autres bistoires, Montréal, Quinze, 1990, p. 172.

3 Marian Engel, Ours, Paris, Calmann-Lévy, 1999, p. 109. Superbe histoire de mots, en l'occurrence, qu'il est impossible de ne pas raconter. Chez les Cary, la propriété de l'île passait de père en fils, à condition que ce dernier fût colonel. L'avant-dernière génération n'eut pas de fils; quand une fille naquit, on la prénomma Colonel, ce qui assurait la continuité de la succession. L'art de raconter connaît bien le rôle que peuvent jouer les mots, comment ils peuvent infléchir l'"histoire", mot éminemment bisémique à tout le moins. Mais toutes ne sont-elles pas, d'abord et avant tout, des "histoires de mots"? 


\section{Premier portrait}

"Portraits d'Elsa" est tout à la fois l'"histoire" éponyme et première du recueil de Marie José Thériault intitulé (et sous-intitulé, en indiquant un générique) Portraits d'Elsa et autres bistoires. Cette histoire, puisque nous continuerons, fidélité lectorale, d'utiliser ce mot qui conte et se raconte, se révèle particulièrement intéressante à plus d'un... titre, dont deux ou trois retiendront ici notre attention.

Tout d'abord, il faut souligner que l'histoire d'Elsa est constituée de six portraits ou tableaux — "D'abord les jambes. Engainées de bas à résille, noirs. "Femme assise" (détail) — comme on le dirait d'un tableau" (p. 13). — qui, selon les divers angles ou regards de la narration, de la caméra ou du miroir, tentent de bien rendre ou peindre Elsa, grâce aux mots qui traduisent ses gestes et ses poses, les étudié(e)s comme les spontané(e)s; les six portraits visant ainsi à en constituer un, complet et crédible si possible, $1 \times 6=1$. Il faut noter ensuite, à la lecture des six portraits, les modifications du mode d'énonciation d'un portrait ou d'une étude à l'autre; enfin, étant donné justement les divers modes d'énonciation, il faut interroger le nom même du sujet/objet des portraits, Elsa, "sa elle", elle selon lui, mais aussi elle selon elle-même, ce dernier "elle" renvoyant à l'autre dont on sait, depuis Rimbaud et Freud au moins, qu'il est aussi l'autre ou les autres $(1 \times 6=1)$ de soi-même.

Les modes d'énonciation, revenons-y, de l'histoire en six portraits. Le premier portrait (et le recueil, donc) commence ainsi : "Quand elle enlève ses bas, Elsa ne les enroule pas jusqu'à la cheville pour les faire ensuite glisser sur le pied. Quand elle enlève ses bas, Elsa les tire par la pointe"; donc, vous dites-vous, portrait brossé selon une narration omnisciente, énoncé provenant d'une troisième personne qui, par définition ou presque, sait et connaît tout depuis tantôt ou toujours, depuis une éternité ou l'autre, qui sait "tire[r] la pointe" de son propos. Jusqu'à ce que, à la fin de ce premier portrait, vous lisiez les deux premières lignes de son avant-dernier paragraphe: "En réalité, le rituel d'Elsa ne m'intéresse guère. Pendant qu'il se déroule, je regarde [...]". Donc, révision forcée de ce que vous aviez cru jusque-là : narration au je homodiégétique d'un "client" ("Je plaque mes paumes sur ses seins insignifiants - et je jouis d'elle. En vitesse. Tout habillé.", p. 13) d'Elsa qui jusque-là la regardait agir, plutôt que narration hétérodiégétique selon l'omniscience. 
86

Le deuxième portrait, bel et bien cette fois, est mené selon une narration qui sait tout; mais qui, ainsi que dans le premier, s'attache au détail, au fragment - la caméra, "l'œil artificiel" (p. 15) de N. fixe beaucoup les jambes, les cuisses d'Elsa (p. 14). Et la narration, si elle est surtout menée selon le point de vue de N., n'exclut pas celui d'Elsa :

Ses jambes, Elsa les garde ainsi, immobiles, pendant que l'œil de la caméra de N. les encadre, pendant qu'il en extrait la scandaleuse beauté. Mais pour Elsa, elles deviennent tout à coup postiches et artificiellement fixées à ses cuisses par le troublant désir d'un homme que seul allume le fragment (p. 14).

Comment ne pas dire que la narration omnisciente est assurée depuis un point de vue littéraire, artistique: le point de vue d'Elsa, ici, fait écrire "fragment" à l'instance narratrice, alors que cette dernière aurait pu utiliser un mot comme "fétichisme" ou une expression comme "pulsion partielle", qui renvoient à la psyché, pour décrire la fixation de N., ou cette fixation selon Elsa. Le sexuel formulé selon le point de vue d'une narration lectorale littéraire, tel semble bien être le sens de la dernière phrase de ce deuxième portrait: "Car il n'y a que là, dans ce solitaire décryptage du tout par la partie, que le plaisir de N. peut approcher ses limites dernières" (p. 15). Ainsi qu'Elsa reconnât son abolition dans le plaisir que prend $\mathrm{N}$. à la fragmentation de sa personne, l'instance narratrice sait bien que "le solitaire décryptage du tout par la partie" constitue le seul type de plaisir que ce même N. peut éprouver.

Troisième portrait. Narration omnisciente encore. Selon le point de vue d'un vieux monsieur. Qui raconte des histoires à Elsa, façon pour lui de mettre en branle son désir. "Elsa en robe de petite fille. Fleur parmi les fleurs [...] Seins menus prisonniers des nids d'abeille et cou fragile cerné de lin contrastant qu'Elsa tient droit comme une étamine en affichant un soupçon de dépit" (p. 16). Point de vue du "vieux monsieur", mêmes petits seins d'Elsa dont le point de vue n'est pas oublié — mais c'est le client qui raconte ses histoires:

Tandis qu'elle se mordille le pouce en l'écoutant, le monsieur lui raconte en détail l'histoire d'une petite fille qui ressemblerait à Elsa, l'histoire d'une petite Elsa pleine d'attentions et de complaisance, toujours disponible au mimodrame, à la mise en scène des fables d'un grand-père curieux de ses fictions intérieures (p. 16). 
Merveilleuse ambiguité de ce "ses" des "fictions intérieures": celles du grand-père ou celles d'Elsa? Ou celles de l'une et de l'autre jouant de celles de l'autre et de l'une? Et encore, comme dans la séquence ou portrait précédent, le point de vue artistique de l'instance narrative: le "fragment" du jeu selon la fixation sexuelle a fait place au "mimodrame" du vieil homme racontant ses histoires, dont la "ponctuation consistera en gestes à chaque fois plus précis et obscènes" (p. 17), sexualité et textualité empruntant l'un à l'autre leurs langages pour raconter leurs histoires.

À compter du quatrième portrait, qui marque le début du deuxième versant de cette histoire en six instantanés ou points de vue, les choses tout à la fois changent et continuent: écrit au "il" comme le précédent, ce portrait donne pour la première fois la parole à Elsa: "Pour vous, lui dit Elsa, je suis un paradis à perdre" (p. 18), première phrase du portrait. Elle raconte une ou ses histoire(s): "Elle parle, elle dit ceci ou cela ou elle dit autre chose, comme une qui ânonnerait un texte appris dont elle embrouillerait parfois l'ordre logique (encore que)" (p. 19). Le texte produit son effet: "Pause. Regard de l'homme. Ses doigts d'elfe musicien rejoignent et frôlent et font chanter çà, là, la blanche peau d'Elsa" (p. 20). À la fin, "Elsa pleure. Comme à chaque fois. Car elle le sait: elle est pour lui toutes les femmes. Et elle n'en sera jamais aucune" (p. 21).

Ce qui mène, volonté de s'appartenir, au début du cinquième portrait: "Le regard d'Elsa est à l'orée de la hargne... [...]. Le regard d'Elsa est dressé à dresser. De même ses gestes. De même ses mots." (p. 21). Gestes (ayant trait à la sexualité, ici) et mots (sexe et texte), même entreprise. Elsa mènera celle-ci à bien: "Le scénario (son scénario) dont Elsa a respecté rigoureusement la moindre didascalie [mots-gestes] prend fin au moment précis où l'homme la quitte." (p. 22). Elle aperçoit alors son "propre reflet dans la glace": "Décidons (licence d'auteur) qu'Elsa se sourit tout d'abord..." (p. 22).

Quand nous lisons alors, incipit du sixième et dernier portrait: "Moi, Elsa, je lui échappe. Alors pour me reconstituer, il me demande: "Parlez-moi de vous", et il note" (p. 23), quand nous lisons ces lignes, la question se pose en une superbe ambiguité: qui est ce "il" dont parle Elsa? Le "il" des portraits antérieurs, le "il/client" après tout parfois nommé N. comme dans narrateur, ou le "il" d'auteur qui se permet aussi des "licences"? Et si ce "il" 
double sinon triple n'en faisait qu'un? Car dans ce dernier portrait écrit au "je", Elsa parle de son "scribe", de "mon copiste (mon créateur?)" (p. 23-24). Mais qui est ce créateur entre narrateur et auteur, d'autant plus qu'il n'est toujours que le copiste de sa créature? "Où gît la vérité? Qui est la vérité?"(p. 24), se demande Elsa, juste avant de constater qu'il et elle, "par des opérations stratégiques organisables à l'infini", ont "tiss[é]" "de très pénélopiennes attaches" (p. 25). Et de conclure, fin de l'histoire intitulée "Portraits d'Elsa":

Mais en dépit de nos liens, au bout du compte, moi, ElsaElsaElsa, entre toutes moi multipliées, je ne sais au juste où vont les préférences de celui qui me transcrit.

Et j'ignore aussi (et j'ignore surtout) lequel de nous est l'autre dans cette singulière conversation où nous sommes seuls, ensemble (p. 25).

Parcours complété, boucle bouclée: la lecture de cette histoire nous a menés d'un premier portrait selon un "je" masculin qui lisait partiellement Elsa selon son désir à lui, au sixième portrait écrit au "je" d'Elsa qui s'interroge sur son identité et veut la découvrir, la transition s'effectuant dans le passage entre les troisième et quatrième portraits. Mais de même que le premier semblait d'abord écrit au "il" alors qu'il l'était au "je", le sixième, écrit au "je", s'interroge sur l'autre, sur le "il" qui avec elle-même tisse son "je". Qui suis-je, qui est je qui est un autre entre... autres, comment se dire ou se traduire, comment les mots, par fragments, peuvent-ils représenter un être, comment un être de mots écrits sur le papier peut-il rendre compte du réel?

Le texte littéraire, jeu éprouvé qui sait jouer de son propre arbitre en toute liberté organisée, ne saurait en demander beaucoup plus qu'à cette histoire en quinze pages racontée par Marie José Thériault. Voilà un texte on ne peut plus rigoureusement construit, et qui permet pourtant au langage, au mot, de manifester la superbe profusion de ses moyens; voilà une histoire qui, s'écrivant, s'interroge sur les procédés de sa propre écriture; une histoire qui, comme Elsa, réfléchit (sur) son identité propre aux fins de revendiquer sa propre multiplicité autonome.

Une histoire qui, déjà, comme incipit éponyme, donne d'entrée de jeu le parcours des histoires du recueil nommé ainsi que l'on sait. Divisé en trois parties, "Portraits d'Elsa", "Le manuscrit annoté par Pétrarque" - ainsi qu'Elsa s'annote elle-même 
pourrait-on dire — et "Cinq histoires orientales" — ainsi que les histoires nombreuses du "Portraits d'Elsa" constituent déjà des histoires selon le procédé des Mille et une nuits orientales -, le recueil fournit en sa dernière histoire ou nouvelle, "Histoire de Didem...", dont la fin ou presque a été donnée en épigraphe à cet article, la même fin (ou interrogation) que celle de la première histoire du recueil: il faut chanter à la fois les droits de Didem-la-femme et ceux du musicien d'Alep, son chanteur ou narrateur (ou scribe, etc.). Où gît la vérité, entre personnage raconté et personnage racontant, les deux ayant partie liée dans toute histoire qui se raconte?

S'agissant d'histoire et de qui la raconte, citons ici les remarques du commentateur (licence d'auteur) qui, à la fin de chaque bobine (chapitre) fait le point (critique) sur les propos tenus par le narrateur du récit. Ces remarques s'inscrivent à la fin de la bobine (chapitre) 12 du roman d'Yves Thériault intitulé Le grand roman d'un petit homme:

Je sens que le terme approche, que vous allez maintenant conclure, Arsène. Je vous crois assez habile pour rattacher la fin de ce qu'il vous reste à dire au début de votre récit, parfaisant ainsi le cercle, ce qui, à votre insu peut-être, est la marque du bon raconteur ${ }^{4}$.

À l'insu d'Arsène peut-être; mais certes pas à l'insu de Marie José Thériault. Ni, sans doute, à l'insu d'Yves-du-Même-Patronyme qui pratiquait déjà, et bien allègrement, la licence comme les interrogations d'auteur.

\section{Deuxième portrait}

Dans une entrevue donnée en février 1987, Marie José Thériault, à qui l'on venait de demander s'il n'était pas "parfois très lourd" de pratiquer soi-même l'écriture alors qu'on était la fille d'Yves Thériault, répondait entre autres choses ce qui suit:

Par conséquent, je craignais beaucoup qu'au moment où je commencerais à écrire, on dirait encore deux fois plus "la fille d'Yves Thériault", et que les comparaisons se multiplieraient. Or, c'est précisément le contraire qui s'est produit: c'est à partir

4 Yves Thériault, Le grand roman d'un petit homme, Montréal, Jour, 1963, p. 131. 
du jour où je suis devenue écrivain que je suis devenue "Marie José Thériault" et qu'on a cessé de dire que j'étais "la fille de". D'autres craintes ont fait que j'ai opté pour la poésie: les personnages que j'affectionnais ressemblaient beaucoup aux personnages de mon père, dans la tendance des Contes pour un bomme seul par exemple, ces personnages plus primitifs des premiers livres, un peu taillés à la hache. J'aimais aussi les atmosphères paysannes sans références géographiques précises, et où l'on retrouve beaucoup une certaine Europe ${ }^{5}$.

Marie José Thériault a pratiqué la critique littéraire au Devoir, entre autres lieux, elle a aussi été directrice littéraire, chez $\mathrm{HMH}$ par exemple. Marie José Thériault est une excellente lectrice, dont l'expertise est bien connue et recherchée: elle a fermement recommandé aux éditions Québec/Amérique, à la lecture du manuscrit de La mauvaise foi, la publication de ce premier roman de l'auteur québécois d'origine manitobaine Gérald Tougas (roman qui allait, quelques mois plus tard, obtenir le prix du Gouverneur général) ${ }^{6}$. L'écriture de Tougas, bien différente de celle d'Yves Thériault, avec ses phrases longues faisant retour sur ellesmêmes, son vaste et savant vocabulaire (ce qui ne veut surtout pas dire pédant), et son narrateur nommé Marcel dans un roman qui est une sorte de recherche d'un temps perdu, ce roman avait de quoi, pour qui a lu Marie José Thériault, attirer l'attention de celle-ci.

Marie José Thériault sait lire: elle a su lire Yves Thériault, elle sait se lire. Yves Thériault, on le sait, s'est toujours décrit comme un conteur, et deux fois plutôt qu'une:

J'ai pourtant écrit quelques bonnes douzaines de fois que j'étais un conteur et entendais le rester. Que je n'étais le prédicant d'aucune doctrine, sauf peut-être celle d'un certain retour à la vraie nature en notre monde. [...]

À vrai dire, je me demande si l'on comprend bien lorsque je me prétends conteur. Sait-on ce que cela signifie? En a-t-on vu légion des conteurs véritables? Sait-on la filiation véritable d'un artisan dans ce genre?

Autrefois, il y a peut-être des milliers d'années, lorsque les tribus erraient dans les grandes étendues désertiques, le soir, autour du feu de camp, il s'est trouvé des hommes qui ont meu-

5 Dans Études littéraires, Québec, vol. 21, n 1, printemps-été 1988, p. 153.

6 Gérald Tougas, La mauvaise foi, Montréal, Québec/Amérique, 1990, 266 p. 
blé les heures oisives, en racontant des hauts faits, des aventures, peut-être bien l'histoire même de la tribu et des êtres la constituant. [...]

Il est évident que j'écris des contes qui sont techniquement meilleurs que mes romans. Et que mes romans ne sont souvent que des contes démesurés ${ }^{7}$.

J'oserai donc dire, alors même que telle n'est pas la perception première qu'on peut avoir de lui, qu'Yves Thériault savait lire, savait se lire - il a aussi pratiqué la chronique littéraire dans divers journaux et magazines, il connaissait, d'instinct maîtrisé, ses forces et ses faiblesses. Faut-il bien s'étonner, histoire de parentèles, qu'entre bons lecteurs qui écrivent, Marie José Thériault ait publié, alors qu'elle assurait la direction littéraire chez HMH, Moi, Pierre Huneau ${ }^{8}$ qui, moins connu que l'Agaguk qui jette peutêtre trop d'ombre sur le reste de sa production littéraire, constitue l'une des plus éclatantes réussites du conteur Thériault, alors qu'on parle de ce texte comme d'un roman et que Thériault l'a plus humblement et plus pertinemment nommé "narration"?

Fils de mes lectures comme Don Quichotte et en ce sens fils d'Yves Thériault, j'admets, comme son père, avoir peu lu la production poétique de Marie José Thériault (encore que) 9 . C'est-àdire que j'en ai lu la majeure partie, mais ne sais trop comment parler du texte poétique. Mais j'ai lu l'entier ou presque de sa prose, après celle de son père, et me sens moins d'incompétence pour en parler brièvement. En particulier pour parler de L'envoleur de chevaux et autres contes ${ }^{10}$, de - bien sûr - Portraits d'Elsa et autres histoires, et des Demoiselles de Numidie, roman ${ }^{11}$.

7 Yves Thériault, Textes et documents, Montréal, Leméac, 1969, p. 35-36.

8 Yves Thériault, Moi, Pierre Huneau, Montréal, HMH, 1976, 135 p.

9 Lire sur ce sujet ce que dit Marie José Thériault des réactions de son père alors qu'elle écrivait de la poésie; dans Études littéraires, op. cit., p. 146-147. Pour bien comparer les parcours respectifs de Marie José et Yves Thérault (au sujet du goût du chant en particulier), il vaut certes la peine de lire à la suite les uns des autres le texte intitulé "Yves Thériault se raconte" dans Textes et documents et les deux interviews de Marie José dans le numéro d'Études littéraires déjà cité. Ajouter à cela les entretiens avec André Carpentier, Yves Thériault se raconte, Montréal, VLB, 1985, 188 p. et l'interview que m'accordait Yves Thériault pour Voix et images, vol. V, n 2, hiver 1980, p. 223-243.

10 Marie José Thériault, L'envoleur de chevaux et autres contes, Montréal, Boréal compact, [1986] 1994, 173 p.

11 Marie José Thériault, Les demoiselles de Numidie, Montréal, Boréal express, 1984, 244 p. Entre autres analyses de ce roman, celle d'André Maindron, 
Commençons par souligner ceci: Marie José Thériault a publié davantage de recueils de poèmes que d'ouvrages en prose. (En terme de pages, cependant, elle a bien davantage publié en prose qu'en poésie.) Et, conteuse d'abord sans doute, elle n'a publié qu'un seul roman. Or, ayant lu forcément, Les demoiselles de Numidie après Les temps du carcajou ${ }^{12}$ par exemple, j'éprouve quelques difficultés - problème de fils, dirait l'autre - à ne pas lire le roman de Marie José dans le sillage de celui d'Yves. Romans de la mer et des surprises que réservent les tréfonds marins de l'originelle thalassa, romans dans lesquels apparaît un vaisseau fantôme; et si on peut lire dans "l'Avertissement" placé tout au début des Demoiselles de Numidie: "La langue de certains chapitres, si elle est archaïque, ne prétend pas respecter les règles en vigueur à une époque donnée" (peut-on ici penser, ne serait-ce qu'un moment, à la langue archaïque du vieux pêcheur narrateur de Moi, Pierre Huneau?), le déroulement des Temps du carcajou montre avec éclat la puissance de l'archaïsme présent en notre psyché, de la passion jalouse et vengeresse du personnage principal, Bruno Juchereau qui, malgré la culture qu'il s'est donnée, reste bien près de personnages primitifs tel celui qu'on retrouve, par exemple, dans "Challu-la-chaîne" des Contes pour un homme seul, premier livre d'Yves publié en 1944.

Archaïsmes différents, plus précisément manifestations différentes de l'archaïsme, mais présence de l'archaïsme dans les deux romans. Renversement fort important de l'un à l'autre roman par ailleurs: dans la vieille rivalité entre les sexes, Juchereau-le-Mâle mène une vengeance de cauchemar; dans Les demoiselles de Numidie, ce sont elles, les femmes-sirènes, qui attirent les marins. Et là où le roman d'Yves utilise son langage le plus direct et le plus impitoyable ("Ma phrase n'est pas une phrase de styliste [...]. C'est une phrase d'utilité. J'aime le mot en tant qu'outil visant à faire vivre...", Textes et documents, p. 27-28), la langue des Demoiselles, "sensuelle et fastueuse", selon la quatrième de couver-

"Serena Klein Todd ou le paradoxe romanesque", dans Voix et images, vol. $\mathrm{XV}, \mathrm{n}^{\circ} 3$, printemps 1990, p. 424-436. Article particulièrement approprié pour nous ici, puisque ce numéro de Tangence porte sur la parentèle et que la lecture des Demoiselles par Maindron s'inspire de l'essai de Marthe Robert, Roman des origines et origines du roman (Paris, Grasset, 1972), essai que nous avons beaucoup fréquenté et qui doit donc d'une manière ou d'une autre influencer le présent article.

12 Yves Thériault, Les temps du carcajou, Paris, Robert Laffont, 1966, 246 p. 
ture, mène un joyeux sabbat qui contraste singulièrement avec le cauchemar du Carcajou.

La mer des Temps du carcajou est le Saint-Laurent et son golfe; celle des Demoiselles de Numidie (voir ce dernier mot dans une bonne encyclopédie) est plutôt l'Atlantique nord au sortir de la Méditerranée depuis les colonnes d'Hercule, c'est-à-dire depuis le détroit de Gibraltar. J'avance l'hypothèse suivante, s'agissant des contes-histoires-nouvelles d'Yves et Marie José, en tenant un compte particulier des remarques de cette dernière quant aux affinités qu'elle savait déceler entre ses goûts à elle et les personnages dits primitifs qu'Yves savait si bien faire naître: l'Orient de plusieurs contes de Marie José, ceux des dernières parties de L'envoleur de chevaux et de Portraits d'Elsa en particulier, cet Orient originel, mystérieux et cultivé constitue pour elle comme l'équivalent de ce que sont, pour Yves, les Indiens et la Côte Nord du Québec, personnages et lieu également originels, autochtones, en Terre du Caïn biblique.

Sauf que le mystère, dans ce dernier cas, relève davantage de la nature, sinon de l'instinct, que de la culture. Dans un texte publié il y a quelques années par la même revue Tangence, j'avais longuement analysé "L'île introuvable", nouvelle première et éponyme d'un recueil d'Yves Thériault ${ }^{13}$; si l'espace le permettait, il faudrait tout à la fois prolonger cette analyse et la placer en regard d'une analyse semblable de la superbe histoire ayant pour titre "Le manuscrit annoté par Pétrarque" qui se trouve au cour et au centre de Portraits d'Elsa. Les parentés et les différences, entre Thériault père et fille, ressortiraient dans l'éclat de leur pénélopien tissage: parentés dans l'appropriation de textes antérieurs (poème de Pierre Perrault pour Yves; manuscrit annoté par Pétrarque pour Marie José), dans l'appropriation par Yves de traditions nordiques et par Marie José de la tradition littéraire orientale, différences dans le mode d'appropriation de ces textes et donc dans le langage des histoires (ou vice versa): la découverte du poème de Perrault s'inscrit dans le déroulement de la vie de tous les jours, le manuscrit annoté devient le point de départ et la fixation du bibliophile narrateur rêvant dès lors de voir son nom associé à une découverte savante qui, en plus, lierait son nom à celui du célèbre auteur italien.

13 Dans Tangence, Rimouski, no 50 (Lectures de nouvelles québécoises), mars 1996, p. 20-35. 
94

Et puisqu'il vient d'être question de Pétrarque, j'avancerai, fin de l'hypothèse, que l'Italie, avec son Nord et son Sud, entre l'Est et l'Ouest, constitue le lieu privilégié où les fissures entre les œuvres d'Yves et de Marie José Thériault peuvent le plus aisément s'abolir, trouver un terrain propice à leur colmatage. Ce sont bel et bien des émigrés italiens qu'Yves, dans Amour au goût de mer ${ }^{14}$ veut intégrer au Québec par Gaspésie interposée, sans trop de résultats; Marie José tente la même expérience d'un point de vue littéraire, en jouant d'un manuscrit annoté par l'amoureux Pétrarque, rencontre d'une histoire québécoise (c'està-dire: d'une histoire racontée par une Québécoise depuis des recherches menées à Troyes en Champagne!) et de l'auteur italien par bibliophile interposé. Arrêtons-nous là; pour l'instant. Question d'espace, nous l'avons dit.

Marie José Thériault sait lire et pratique une écriture "sensuelle et fastueuse", donc. Si Yves Thériault, en ses débuts à la radio, pouvait se féliciter d'être bilingue depuis sa toute première enfance $^{15}$, ce qui veut dire en ce pays qu'on connaît (maîtrise?) les deux langues officielles, l'anglais et le français, Marie José, elle, pratique la traduction de l'anglais au français. Et cette pratique, littéraire entre autres dans son cas, lui a valu à deux reprises le prix du Gouverneur général. Traduire: c'est-à-dire d'une part lire l'Autre dans la langue qui est sienne, et savoir d'autre part l'écrire dans sa langue à soi. On ne saurait mieux dire que l'instance traductrice doit tout à la fois savoir lire et écrire.

Parmi les ouvres littéraires par elle traduites, retenons-en d'abord deux. Pour des raisons ayant trait à la parentèle précisément, c'est-à-dire à la parenté qui peut tisser ses liens, même en cette entreprise, entre les goûts de l'instance traductrice et ceux du parent en écriture. Quitte à parler ensuite d'une troisième traduction qui renvoie plus particulièrement à la pratique d'écriture propre à la seule dite instance (encore que).

On peut lire en français, grâce à la traduction de Marie José Thériault, À l'aube de lendemains précaires, recueil de nouvelles

14 Yves Thériault, Amour au goût de mer, Montréal, Beauchemin, 1961, 132 p. À quoi il faut ajouter les contes "italiens" des divers recueils d'Yves et ce que dit Marie José de l'Italie dans les interviews qu'on peut lire dans Études littéraires, op. cit., et dans son roman Les demoiselles de Numidie.

15 Voir Textes et documents, op. cit., p. 16. 
de l'auteur canadien d'origine trinidadienne Neil Bissoondath ${ }^{16}$. La formule est bien connue, qui a souvent été utilisée par la critique: Yves Thériault, écrivain des minorités. Or le recueil de Bissoondath illustre particulièrement ce statut, celui du minoritaire, selon les situations et les points de vue les plus divers, attendus ou inattendus. Et puis et surtout en ce qui touche ce fils de ses lectures, Marie José Thériault a traduit Bear (1976) de la romancière canadienne Marian Engel, traduction parue en cet été 1999 sous le titre Ours.

Je ne sais plus, mais il me semble que oui forcément, si Marie José et moi n'avons pas discuté il y a quelques années de ce roman de Marian Engel. Et je n'ose le lui demander au moment d'écrire ces lignes. [Encore que: ayant achevé ce texte, je l'ai fait lire à Marie José avant sa publication; lectrice de la qualité que l'on sait, elle m'a fait remarquer que nous avions parlé du roman d'Engel parce qu'elle était engagée dans sa traduction.] Je sais seulement que j'ai lu le roman de Marian Engel au moment de sa parution ou presque pour une raison bien simple, évidente pour un lecteur d'Yves Thériault: celui-ci avait fait paraître en 1968 un bref roman intitulé Kesten ${ }^{17}$. Dont le sujet peut se résumer ainsi: la compagne de Kesten, Ingrid, tombait amoureuse du cheval, Dragon, que son compagnon avait acheté afin de le dompter. Nonobstant Pasiphaé, mère de Phèdre, et le taureau avec lequel elle devait engendrer le Minotaure, les amours entre humains et animaux sont relativement rares en littérature. Or, Ours de Marian Engel met en scène l'héroïne du roman et l'ours dont elle devient l'amante: comment un lecteur de Thériault aurait-il pu n'être pas intéressé à ce roman, comment Marie José, elle-même intéressée

16 Neil Bissoondath, À l'aube de lendemains précaires, Montréal, Boréal, 1994, $311 \mathrm{p}$.

17 Yves Thériault, Kesten, Montréal, Jour, 1968, 123 p. Peut-être est-ce le lieu de suggérer la lecture de Le choix de Marie José Thériault dans l'œuvre d'Yves Thériault (Charlesbourg, Presses laurentiennes, 1986, 79 p.); pour le choix des textes, cela va de soi (où figure un extrait de Kesten), mais aussi pour l'"Avant-propos" qui l'accompagne. Il ne peut guère être indifférent à un lecteur que Marie José décrive son père comme un "ours" (ainsi qu'elle le fait aussi dans Études littéraires, op. cit., p. 143-144); comme un ours tendre qui protégeait sous l'ours sa tendresse et sa vulnérabilité. Faut-il ajouter qu'Yves a écrit des romans intitulés Le dompteur d'ours (Montréal, Cercle du livre de France, 1951) et La quête de l'ourse (Montréal, Stanké, 1980) et qu'un ours totémique joue un rôle fort important dans Tayaout, fils d'Agaguk (Montréal, l'Homme, 1969). 
par les personnages primitifs créés par son père, comment l'un et l'autre auraient-ils pu ne pas lire le roman d'Engel? [Or, me dit Marie José, elle n'a jamais pensé à Kesten au moment de traduire Bear.] Quitte à constater que, du roman de Thériault à celui d'Engel, l'action passe d'une grande violence sexuelle à une action discrète et sensuelle, délicate (ainsi que, pourrait-on dire, on passe du Carcajou aux Demoiselles de Numidie).

Une troisième traduction, celle de L'œuvre $d u$ Gallois de Robert Walshe, romancier originaire de la Saskatchewan ${ }^{18}$. Roman complexe, difficile, à la Nabokov, Borges ou Aquin. Roman qui convoque lui aussi Pétrarque (p. 251) et met en œuvre des moyens littéraires hautement sophistiqués et un langage approprié qui ne vont pas sans jouer des mêmes cordes que celles dont aime jouer l'auteure du "Manuscrit annoté " déjà cité ou du "Livre de Mafteh Haller" de L'envoleur, "Livre" qui déjà jouait des mêmes accords que le "Manuscrit". Née forcément dans la parentèle d'Yves, Marie José traductrice accorde ici sa musique personnelle. Celle qu'elle a créée afin d'assurer son autonomie, sa "poésie", pour reprendre le nom de sa première pratique littéraire, mais en un sens bien plus vaste que le genre qu'il désigne

\section{Trousième portrait}

Conteuse, fille de conteur déclaré, Marie José Thériault est aussi la fille de sa mère Michelle. De Germaine-Michelle Blanchet, sœur du chansonnier-compositeur Jacques Blanchet, auteur entre autres de la célèbre chanson intitulée "le Soleil se marie avec la mer". (Parentèle, parentèle: le même Jacques Blanchet signa le décor lors de la création du Marcheur d'Yves Thériault au Gesù en 1950; et ce fut J.-Alcide Thériault, père d'Yves et menuisier de son métier, qui réalisa ledit décor ${ }^{19}$.) Dans Textes et documents, Yves Thériault avait déjà signalé les ressources d'écrivain de son épouse, racontant comment, à la fin des années 1940, ils écrivaient l'un et l'autre sous divers pseudonymes des romans à dix cents (p. 20-21). Dans une entrevue avec Sophie Stanké en 1984, Marie José répond à l'intervieweuse qui l'interroge sur le rôle jouée par sa mère auprès d'elle et de son père :

18 Robert Walshe, L'ouvre du Gallois, Montréal, Boréal, 1993, 450 p.

19 Yves Thériault, Le marcheur, Montréal, SYT, 1996, p. 31. 
Eh bien, comme toutes les mères, elle a d'abord été la mère... Maintenant, elle est aussi l'amie. Pour ce qui est de mon père, elle a été très importante dans sa carrière, elle l'a beaucoup soutenu, elle l'a beaucoup encouragé au début, elle l'a aidé, elle a collaboré avec lui. Même tout au début, quand il écrivait des "romans à dix cents", payés à la page, pour vivre, maman en écrivait presque autant que lui par jour et par semaine; à eux deux, ils arrivaient à faire vivre la maisonnée, ce qui n'était pas tous les jours facile. Donc, elle l'a beaucoup aidé.

À l'intervieweuse qui insiste, qui dit: "c'est quelqu'un qui a été très très... 80\% d'Yves Thériault peut-être", Marie José répond:

Bien entendu, le talent de Thériault, c'est lui qui l'avait; l'imagination de Thériault, c'est lui qui l'avait. Maman l'a aidé à mettre tout ça en ordre; elle apportait un travail de finition; elle le dirigeait un peu dans son écriture; elle l'a aussi amené à croire en lui, surtout au début, et même après ses premiers livres, parce qu'il était rempli de doutes (que cela ait été évident ou non) comme la plupart des écrivains qui commencent et même qui continuent ${ }^{20}$.

Les parentés semblant décidément de rigueur, peut-être faut-il, sur ce chapitre, laisser le dernier mot à Alain Stanké :

En plus d'être son critique le plus juste et le plus sévère, Michelle, la femme d'Yves, était sa plus précieuse collaboratrice. Grâce à sa parfaite connaissance du français, elle relisait et corrigeait tout ce que son mari écrivait. Le jour où Yves m’annonça que le couple se séparait, je fus très peiné. J'aimais les voir tous deux vivant harmonieusement et se complétant parfaitement dans le travail. Tous les proches des Thériault pourraient en témoigner. N'eût été de son épouse, Yves n'aurait probablement jamais atteint la renommée qu'il a connue. Reconnaissant que sa femme était la seule capable de peaufiner son écriture, en même temps qu'il m'annonça la triste nouvelle il me fit part de son inquiétude:

- Il va falloir que tu trouves quelqu'un d'aussi bon que Michelle. Ça ne va pas être facile ${ }^{21}$ !

20 Dans Études littéraires, op. cit., p. 144.

21 Alain Stanké, Occasions de bonheur, Montréal, Stanké, 1993, p. 46-47. Histoire de mots (suite): Stanké parle aussi longuement et chaleureusement de Gabrielle Roy, l'auteure de Bonheur d'occasion, dans son livre; et j'ajouterai qu'Yves Thériault avait un respect particulier pour l'œuvre de la romancière d'origine manitobaine. 
Il faut lire aussi la suite de ces lignes de Stanké : comment, à bout de ressources, il finit par demander à Michelle de continuer à être l'editor des textes d'Yves, mais sans que ce dernier le sache. Et comment Thériault, trouvant de si grande qualité, si "bonne" sa nouvelle lectrice-correctrice, insistait auprès de Stanké, qui ne flancha pas, pour qu'elle lui fût présentée!

Le même Alain Stanké publia, presque à la fin de la vie de Michelle, un recueil de nouvelles de cette dernière, L'écume des choses $^{22}$. En quatrième de couverture de ce recueil, il souligne le rôle discret et effacé, mais combien important qu'elle a joué dans bien des carrières littéraires: "... pendant qu'elle faisait ce qu'elle savait faire le mieux, ses auteurs étaient interviewés, photographiés, encensés".

"Maintenant, elle est aussi l'amie ", disait Marie José parlant de sa mère en 1984. Depuis Gérard Genette et ses Seuils ${ }^{23}$ en particulier, nous savons l'importance qu'il faut accorder au péritexte. Cela étant, il faut certes accorder l'importance nécessaire aux faits suivants: dans Lettera amorosa ${ }^{24}$, recueil de poèmes de Marie José, les illustrations sont de Michelle (ainsi que jadis, en 1960, Ashini $^{25}$ d'Yves contenait la reproduction d'une huile de $\mathrm{Mi}$ chelle); l'une des premières traductions de Marie José, Rocky de Julia Sorel ${ }^{26}$, fut effectuée en collaboration avec sa mère; on peut lire, tout au début de Portraits d'Elsa, que "l'auteur tient à remercier Michelle Thériault pour sa précieuse collaboration"; enfin, il y a ce petit (forcément) livre de Marie José Thériault intitulé $L e$ petit Thériault ${ }^{27}$. Dont le titre complet se continue ainsi : 365 dictées, colles et pièges en capsules pour apprentis champions pressés. C'est Yves qui aurait été content, lui qui admettait, dans Textes et documents, avoir, en ses débuts en écriture, beaucoup souffert de

22 Michelle Thériault, L'écume des choses, Montréal, Stanké, 1994, 155 p. Histoire de mots (suite bis) : et L'écume des jours, dirait Boris Vian?

23 Gérard Genette, Seuils, Paris, Seuil, 1987, 394 p.

24 Marie José Thériault, Lettera amorosa, Montréal, HMH, 1978, 89 p.

25 Yves Thériault, Ashini, Montréal, Fides, 1960, 173 p.

26 Julia Sorel, Rocky (traduction de Marie José Thériault en collaboration avec Michelle Thériault), Montréal et Paris, Quinze et Oyez, 1977, 192 p.

27 Marie José Thériault, Le petit Thériault, Montréal, Fortuna, 1989, 123 p. La maison d'édition de ce livre, Fortuna, est l'occasion de rappeler le nom de cette autre maison d'édition ayant la même adresse postale, les Éditions Sans Nom, création de Marie José qui lui permit de publier Rencontres/Encuentros (1989), recueil de nouvelles bilingue illustré Argentine-Québec. 
son "ignorance sur le plan de la langue" et avoir, dès lors, "inventé un style" qui visait à contourner "les pièges des temps de verbe trop compliqués ou les constructions syntaxiques trop complexes" (p. 11)! Michelle veillait sur Yves, Michelle veille aussi sur Le petit Thériault dans lequel on peut lire (p. 14): "Les dictées suivies d'un astérisque sont de Michelle Thériault". Je n'ai pas fait le compte des *, n'étant pas comptable, mais je sais seulement que les dictées n'étaient pas le fort d'Yves, non plus peut-être qu'une histoire comme "Portraits d'Elsa" (encore que. Cet "encore que" renvoyant aux Commettants de Caridad $^{28}$, roman qui sait écrire une même histoire depuis des points de vue différents). Lecteur de la parentèle Thériault en train d'écrire mes lectures, je sais bien ce que je suis sur le point d'écrire: que l'écriture de Marie José relève tout à la fois de ses affinités avec les personnages primitifs des Contes de son père et du travail peaufiné de la langue pratiqué par sa mère. Et puis-je ajouter qu'il me semble avoir reconnu le "personnage" de Marie José à la fois dans l'Angèle de Moi, Pierre Huneau et dans la Sophie de la nouvelle éponyme, la dernière du recueil, de L'écume des choses? Ce ne serait que justice: les Croyants de l'art de raconter ne doivent jamais cesser de faire chanter leurs histoires. Pour leur enchantement, celui de leurs fidèles et de tous leurs descendants.

\section{Épilogue}

Parentèle, souvenir. Je me souviens de ce coup de téléphone de Marie José Thériault, il y a deux ans environ, alors qu'elle était à la rédaction, qui se poursuit, de la biographie d'Yves: "Tu sais où j'en suis rendue, Renald? Je viens de marier Papa et Maman". Merveilleux, qui peut demander plus? Telle est bien l'une des vertus des conteurs, celle de la re-créer, l'histoire, et de la remettre au monde. Et je m'avise, au moment de conclure, que Maurice Émond a consacré au père un livre et, une vingtaine d'années après, à la fille un article ${ }^{29}$. Ah! la parentèle.

28 Yves Thériault, Les commettants de Caridad, Québec, Institut littéraire du Québec, 1961, 300 p.

29 Maurice Émond, Yves Thériault et le combat de l'homme, Montréal, HMH, 1973, 170 p.; mais aussi "Lecture thématique d'un roman fantastique: Les demoiselles de Numidie de Marie José Thériault ", dans Louise Milot et Jaap Lintvelt (dir.), Le roman québécois depuis 1960. Méthodes et analyses, Sainte-Foy, Presses de l'Université Laval, 1992, p. 19-34. 